ten, die mit dem Asthmamedikament behandelt wurden, war das Risiko, in den nachfolgenden elf Jahren an Parkinson zu erkranken, fast um ein Drittel niedriger als in der Gesamtbevölkerung. Umgekehrt war das PK-Risiko bei den zirka 9.000 Patienten, denen der Betablocker Propanolol verordnet worden war, mehr als doppelt so hoch [Mittal S et al. Science 2017; 357: 891-8. doi: 10.1126/science.aaf3934]. Volkmann warnte jedoch vor verfrühter Euphorie. „Bevor man das Asthmamedikament bei Parkinson-Patienten einsetzt, muss deren Nutzen in großen prospektiven Studien untersucht werden."

\section{Hauttest zur Frühdiagnose}

Diese spannenden Erkenntnisse werfen Fragen zu einer möglicherweise prophylaktischen Parkinson-Therapie auf, etwa bei PK-Risikopatienten. Da die Anfangssymptome in prodromalen PK-Stadien meist sehr unspezifisch sind (Obstipation, Riechstö- rung, Angst, Depression) wäre es laut Volkmann wünschenswert, diagnostische Marker zu entwickeln, mit denen sich die Erkrankung noch vor Beginn der motorischen Symptome aufspüren ließe. Denkbar wäre eine Hautbiopsie als Screening-Instrument. Denn bei mehr als jedem zweiten PK-Risikopatienten (55\%) mit sogenannten REM-Schlafverhaltensstörung konnten deutsche Neurowissenschaftler bereits Jahre vor der klinischen Manifestation der Erkrankung den Biomarker a-Synuklein auch in der Haut nachweisen [Doppler K et al. Acta Neuropath 2017; 133: 535-45]. Die frühe Identifizierung dieser Patienten, so Volkmann, sei wichtig, um sie entsprechenden Studien zuführen zu können, in denen krankheitsmodifizierende Therapien getestet werden.

Dr. Martina-Jasmin Utzt

Fachpressekonferenz "Neurologische Forschung im Fokus", 90. Kongress

der Deutschen Gesellschaft für Neurologie (DGN), Leipzig, 22.9.2017

\title{
Seltene neurologische Erkrankungen zunehmend behandelbar
}

\author{
Seltene neurologische Erkrankungen haben oft \\ genetische Ursachen, die heute mehr und mehr \\ durch spezifische molekulare Therapien behandelt \\ werden können. Als Meilenstein gilt die Entwicklung \\ des Medikamentes Nusinersen zur Behandlung der \\ spinalen Muskelatrophie.
}

\begin{abstract}
„Die Neurologie ist eine Domäne der seltenen Erkrankungen“, erklärte Professor Christine Klein, Direktorin des Instituts für Neurogenetik am Universitätsklinikum Lübeck. Denn $80 \%$ der bekannten 5.000 bis 6.000 seltenen Erkrankungen (SE) weisen eine Beteiligung des peripheren oder zentralen Nervensystems auf. Wiederum $80 \%$ haben eine genetische Ursache, die sich immer häufiger identifizieren lässt und damit den ersten Schritt in Richtung möglicher Therapie darstellt.
\end{abstract}

\section{Oft lange diagnostische Latenz - aber auch steigende Aufklärungsquoten}

Während SE-Patienten noch bis vor kurzem häufig lange ArztOdysseen auf sich nehmen mussten, mit Aufklärungsraten von maximal 5\%, können heute dank moderner Sequenziermethoden knapp $30 \%$ der Fälle aufgeklärt werden. „Viele Patienten sind schon dankbar, wenn ihre Erkrankung einen Namen hat", betonte Klein. Bei der neurogenetischen Diagnostik müsse man allerdings „noch viel mehr als bisher über den Tellerrand schauen“. Denn etwa $5 \%$ der SE-Patienten leiden an zwei unterschiedlichen genetischen Erkrankungen, teils mit phänotypischen Überlappungen. In rund $70 \%$ der Fälle wird die Erkrankung durch Neumutationen ausgelöst, sodass die betroffenen Patienten nicht durch eine positive Familienanamnese als wichtiges Erkennungszeichen für Erbkrankheiten auffallen. Darüber hinaus kann die Penetranz genetischer Mutationen unterschiedlich sein, wie Klein am Beispiel von LRRK2-Mutationen bei genetischem Parkinson-Syndrom ausführte. Dass man mit relativ einfachen Mitteln bei SE oft viel bewegen kann, schilderte Klein am Fall einer jungen Patientin mit Dopa-responsiver Dystonie, die sich als bizarre Bewegungsstörung manifestierte. Bereits nach kleinen Dosen von L-Dopa zeigte die Patientin ein annähernd normales Gangbild, die Erkrankung „verschwand wie durch Zauberhand“. Dennoch beträgt die durchschnittliche diagnostische Latenz noch immer mehr als 13 Jahre. „Das ist natürlich eine Katastrophe angesichts einer Erkrankung, die mithilfe eines Gentests leicht diagnostizierbar und gut behandelbar ist!“

\section{Antisense-Therapie: Durchbruch bei SMA}

Bis dato können erst $10 \%$ der SE spezifisch therapiert werden. „Wir sind aber auf dem richtigen Weg“, ist Klein überzeugt. Wichtig sei es, Erkenntnisse aus der Grundlagenforschung schnellstmöglich zum Patienten zu bringen. In der Behandlung der spinalen Muskelatrophie (SMA) ist dies vor kurzem gelungen. Das im Juni 2017 zugelassene Medikament Nusinersen verlangsamt das Fortschreiten dieser neuromuskulären Erbkrankheit. Die Therapie zielt darauf ab, das mutierte Exon mithilfe molekularer Techniken aus dem betroffenen Gen herauszuschneiden. Sogenannte Antisense-Oligonukleotide (ASOs) blockieren gezielt bestimmte Abschnitte der Boten-RNA. Dadurch kann ein annähernd intaktes Protein hergestellt werden, das zumindest Teilfunktionen übernehmen kann. US-amerikanische Studien hätten bemerkenswerte Erfolge gezeigt. SMA-Kinder, die zuvor beatmet werden mussten, konnten sechs Monate nach Therapiebeginn erstmals sitzen. Die Antisense-Therapie, so Klein, ist auch für andere seltene Erbkrankheiten eine vielversprechende Option. Derzeit laufen unter anderem Therapiestudien bei der Huntington-Krankheit. Einziger Wermutstropfen seien die derzeit hohen Jahrestherapiekosten von etwa einer halben Million $€$. Initiativen wie das 2010 vom Bundesministerium für Gesundheit (BMG) gegründete „Aktionsbündnis für Menschen mit Seltenen Erkrankungen“ (NAMSE) sollen die Versorgung betroffener Patienten verbessern.

Dr. Martina-Jasmin Utzt

Fachpressekonferenz "Neurologische Forschung im Fokus", 90. Kongress der Deutschen Gesellschaft für Neurologie (DGN), Leipzig, 22.9.2017 